\title{
Near-Capacity Iteratively Decoded Space-Time Block Coding
}

\author{
Soon Xin $\mathrm{Ng}^{1}$, Sushanta Das ${ }^{2}$, Jin Wang ${ }^{1}$ and Lajos Hanzo ${ }^{1}$ \\ ${ }^{1}$ School of Electronics and Computer Science, University of Southampton, SO17 1BJ, United Kingdom. \\ Email: $\{$ sxn,lh\}@ecs.soton.ac.uk \\ ${ }^{2}$ University of Texas, Dallas (currently with Philips Research North America, Briarcliff Manor, NY, USA). \\ Email: sushanta.das@philips.com
}

\begin{abstract}
Iteratively decoded near-capacity Space-Time Block Coding (STBC) schemes are designed. Recursive unity-rate codes and IRregular Convolutional Codes (IRCCs) are employed for assisting the conventional non-recursive STBC schemes in achieving decoding convergence to an infinitesimally low bit error ratio at near-capacity signal-to-noise ratios. IRregular Convolutional Codes (IRCCs) are used as the outer codes for achieving a near-capacity performance. It was shown that the resultant iteratively decoded STBC schemes are capable of approaching the corresponding channel capacity within $0.4 \mathrm{~dB}$, when communicating over uncorrelated, flat Rayleigh fading channels.
\end{abstract}

\section{INTRODUCTION}

Information theoretic analysis by Foschini [1] and Telatar [2] shows that a Multiple-Input Multiple-Output (MIMO) scheme, which employs multiple antennas at both the transmitter and the receiver enables high-throughput wireless communication. Space-time codes, introduced by Tarokh et al. [3], constitutes a transmit diversity approach that is capable of improving the integrity of transmissions over fading channels by exploiting the correlation of the transmitted information across different transmit antennas and time slots. Another attractive transmit diversity approach is represented by Space-Time Block Coding (STBC) schemes [4], [5], where full diversity is achieved with the aid of symbol-by-symbol maximum-likelihood decoding algorithm at the receiver. However, it was shown in [5] that no complex-valued orthogonal design based STBC scheme exists, which is capable of achieving a full transmit-diversity gain and a full transmission rate $^{1}$ for more than two transmit antennas. Hence, 'partial-rate' and 'partial-diversity' based STBC schemes have been designed in [5] and [6], respectively. In order to circumvent the abovementioned limitations of linear STBC, recently, non-linear full-rate and full-diversity STBC schemes were proposed for four transmit antennas in [7], [8].

EXtrinsic information transfer (EXIT) charts have recently emerged as a useful tool for analysing the convergence properties of iterative decoding aided concatenated coding schemes [9]. As an advantage, this can be achieved without performing time-consuming Monte-Carlo simulations. The information provided by EXIT charts can be used for finding powerful codes exhibiting guaranteed convergence for a given channel condition. Specifically, near-capacity codes have been successfully designed by applying an EXIT-chart-based technique in [10], [11]. Tüchler and Hagenauer [11], [12] proposed the employment of IRregular Convolutional Codes (IRCCs), which are constituted by a family of convolutional codes having different code rates. They were specifically designed with the aid of EXIT charts [9], for the sake of improving the convergence behaviour of iteratively decoded systems. It was also concluded in [13] that for a three-stage serially concatenated system a recursive Unity-Rate Code (URC), should be employed at the intermediate encoding/decoding stage in order to achieve optimal decoding convergence. In this contribution, a near-capacity three-stage serially concatenated STBC, URC and IRCC scheme was designed with the aid of EXIT charts.

The financial support of the EPSRC UK and that of the European Union is gratefully acknowledged.

${ }^{1}$ A full transmission rate refers to $\log _{2}(\mathcal{M})$, where $\mathcal{M}$ is the number of modulation levels.
The rest of the paper is organized as follows. In Section II, we outlined our preliminary system model and the background of STBC, URC and IRCC schemes. In Section III, our design is characterized based on the channel capacity with the aid of EXIT charts. Our simulation results are presented in Section IV, while our concluding remarks are given in Section V.

\section{Preliminaries}

In this paper, (.) denotes the complex conjugate transpose or complex conjugate of (.), if (.) is a complex-valued matrix or a complex scalar, respectively. Furthermore, $\|()\|,.(.)^{T}$ and (.) denote the Frobenius norm, the transpose and the vector of (.), respectively. Boldface lower case letters will be used to denote a complex-valued scalar, while boldface upper case letters will be used to denote a complex-valued matrix. The notation $\mathbf{a}(j)$ and $\mathbf{a}_{(i, j)}$ denote the $j$ th column and the $(i, j)$ th element of matrix $\mathbf{A}$, respectively.

\section{A. System Model}

We consider a quasi-static flat-fading channel, where we transmit information coded over $N_{t}$ transmit antennas and the receiver is equipped with $N_{r}$ receive antennas. We assume that the transmitter has no channel state information (CSI), whereas the receiver is capable of perfectly tracking the channel. The space-time block code is designed for encoding over $V=N_{t}$ transmission symbols and provided that the channel's envelope may be considered quasi-static over this period, the received signal after demodulation can be written as:

$$
\mathbf{Y}=\mathbf{H X}+\mathbf{W},
$$

where $\mathbf{Y}=[\mathbf{y}(1), \ldots, \mathbf{y}(V)] \in \mathbb{C}^{N_{r} \times V}$ is the sampled received signal matrix, $\mathbf{H} \in \mathbb{C}^{N_{r} \times N_{t}}$ is the quasi-static channel matrix, which is constant over $V$ symbol periods, $\mathbf{X}=[\mathbf{x}(1), \ldots, \mathbf{x}(V)] \in \mathbb{C}^{N_{t} \times V}$ is the STBC transmitted signal matrix and $\mathbf{W}=[\mathbf{w}(1), \ldots, \mathbf{w}(V)] \in$ $\mathbb{C}^{N_{r} \times V}$ represents the additive white Gaussian noise having a variance of $N_{0} / 2$ per dimension.

\section{B. STBC for Two and Four Transmit Antennas}

The design is applicable to all STBC schemes, but we only consider STBC schemes employing $N_{t}=2$ and $N_{t}=4$ transmit antennas in this paper. A complex-valued orthogonal space-time block code using $N_{t}=2$ was discovered by Alamouti [4], which is described by a $(2 \times 2)$ matrix, where the columns represent different time slots, while the rows represent different antennas, and the entries are the symbols to be transmitted. The two complex-valued information symbols, namely $\mathbf{x}_{1}$ and $\mathbf{x}_{2}$, are encoded using Alamouti's $G_{2}$ code as follows:

$$
\mathbf{X}_{G_{2}}=\left(\begin{array}{cc}
\mathbf{x}_{1} & \mathbf{x}_{2} \\
-\overline{\mathbf{x}}_{2} & \overline{\mathbf{x}}_{1}
\end{array}\right)^{T} .
$$

The $G_{2}$ code is a full-rate and full-diversity STBC scheme. We may substitute $\mathbf{X}=\mathbf{X}_{G_{2}}$ into Eq. (1) for the $N_{t}=V=2$ scenario. Jafarkhani extended the $G_{2}$ code into a quasi-orthogonal 
code, namely $Q_{O}$, in order to achieve a full-rate and a transmit diversity order of $N_{t} / 2$ for $N_{t}=V=4$ in [6]. Upon denoting the matrix in Eq. (2) by $\mathbf{A}_{12}$, where the subscript 12 denotes the indices of the two information symbols $\mathbf{x}_{1}$ and $\mathbf{x}_{2}$, we may represent the $Q_{O}$ code of [6] as follows ${ }^{2}$ :

$$
\begin{aligned}
\mathbf{X}_{Q_{O}}= & \left(\begin{array}{cc}
\mathbf{A}_{12} & \mathbf{A}_{34} \\
-\overline{\mathbf{A}}_{34} & \overline{\mathbf{A}}_{12}
\end{array}\right)^{T} \\
= & \left(\begin{array}{cccc}
\mathbf{x}_{1} & \mathbf{x}_{2} & \mathbf{x}_{3} & \mathbf{x}_{4} \\
-\overline{\mathbf{x}}_{2} & \overline{\mathbf{x}}_{1} & -\overline{\mathbf{x}}_{4} & \overline{\mathbf{x}}_{3} \\
-\overline{\mathbf{x}}_{3} & \mathbf{x}_{4} & \overline{\mathbf{x}}_{1} & -\overline{\mathbf{x}}_{2} \\
-\overline{\mathbf{x}}_{4} & -\mathbf{x}_{3} & \mathbf{x}_{2} & \mathbf{x}_{1}
\end{array}\right)^{T} .
\end{aligned}
$$

Calderbank et al. noted that the $G_{2}$ code constitutes a matrix representation of a so-called quaternion scalar and designed a nonlinear full-rate, full-diversity code for $N_{t}=V=4$, namely $Q_{4}$, defined as [8]:

$$
\mathbf{X}_{Q_{4}}=\left(\begin{array}{cc}
\mathbf{A}_{12} & \mathbf{A}_{34} \\
-\overline{\mathbf{A}}_{34} & \frac{\overline{\mathbf{A}}_{34} \overline{\mathbf{A}}_{12} \mathbf{A}_{34}}{\left|\mathbf{x}_{3}\right|^{2}+\left|\mathbf{x}_{4}\right|^{2}}
\end{array}\right)^{T} .
$$

More explicitly, the $Q_{4}$ code is non-linear due to the noncommutative property of quaternions. However, the non-linear part of the code, namely the $(2 \times 2)$ matrix at the bottom right corner of Eq. (5), does not result in any constellation expansion for 4-ary Quadrature Amplitude Modulation (4QAM).

\section{Recursive Unity Rate Codes}

It was shown in [13], [14] that a recursive code is needed as an intermediate code, when the inner code is non-recursive, in order to achieve decoding convergence at low Signal-to-Noise Ratios (SNR). From our EXIT chart analysis, we found that a minimum Hamming distance of two is needed for an inner code in order to achieve decoding convergence at low SNR and a recursive code has at least a minimum Hamming distance of two. We note that there is experimental evidence that a simple recursive URC having a unit memory is capable of providing a minimum Hamming distance of two without reducing the effective throughput. The URC employed has a generator polynomial of $\frac{1}{1+D}$ and it is used as an intermediate code between the IRCC and STBC schemes. A modulo- $2^{B}$ adder is used in the URC scheme, where $B$ is the number of bits per URC symbol. We have $B=1$ for a binary URC and $B>1$ for symbolbased URCs [14].

\section{Irregular Convolutional Code}

An IRCC is constructed from a family of $P$ subcodes. First, a rate- $r$ convolutional mother code $C_{1}$ is selected and the $(P-1)$ other subcodes $C_{k}$ of rate $r_{k}>r$ are obtained by puncturing. Let $L$ denote the total number of encoded bits generated from the $K$ uncoded information bits. Each subcode encodes a fraction of $\alpha_{k} r_{k} L$ of the original uncoded information bits and generates $\alpha_{k} L$ encoded bits. Given the target overall average code rate of $R \in[0,1]$, the weighting coefficient $\alpha_{k}$ has to satisfy:

$$
1=\sum_{k=1}^{P} \alpha_{k}, R=\sum_{k=1}^{P} \alpha_{k} r_{k}, \text { and } \alpha_{k} \in[0,1], \forall k .
$$

Clearly, the individual code rates $r_{k}$ and the weighting coefficients $\alpha_{k}$ play a crucial role in shaping the EXIT function of the resultant

\footnotetext{
${ }^{2}$ The original $Q_{O}$ code involves only the complex conjugate but not the transpose of the $(2 \times 2)$ matrices $\mathbf{A}_{12}$ and $\mathbf{A}_{34}$. However, they have identical performance.
}

IRCC. For example, in [12] a family of $P=17$ subcodes were constructed from a systematic, rate-1/2, memory-4 mother code defined by the generator polynomial $\left(1, g_{1} / g_{0}\right)$, where $g_{0}=1+D+D^{4}$ is the feedback polynomial and $g_{1}=1+D^{2}+D^{3}+D^{4}$ is the feedforward one. Higher code rates may be obtained by puncturing, while lower rates are created by adding more generators and by puncturing under the constraint of maximising the achievable free distance. In the proposed system the two additional generators are $g_{2}=1+D+D^{2}+D^{4}$ and $g_{3}=1+D+D^{3}+D^{4}$. The resultant 17 subcodes have coding rates spanning from $0.1,0.15,0.2, \cdots$, to 0.9 .

The EXIT function of an IRCC can be obtained from those of its subcodes. More specifically, the EXIT function of the target IRCC is the weighted superposition of the EXIT functions of its subcodes [12]. Hence, a careful selection of the weighting coefficients $\alpha_{k}$ could produce an outer code EXIT curve that matches closely the inner code EXIT curve. When the area between the two EXIT curves is minimized, decoding convergence would be achieved at the lowest possible SNR.

\section{SySTEM DESIGN}

The block diagram of the IRCC-URC-STBC scheme is shown in Fig. 1, where the iterative extrinsic information exchange is between the IRCC decoder and the URC-STBC decoder. In the context of discrete-amplitude QAM [15] and Phase-Shift Keying (PSK) [15] modulation, we encounter a Discrete-input Continuous-output Memoryless Channel (DCMC) [15]. In order to design a near-capacity coding scheme, we first derive the DCMC capacity formula for STBCs in Section III-A. Then, the EXIT chart based code design will be performed in Section III-B.

\section{A. Capacity of STBCs}

When complex-valued $\mathcal{M}$-ary PSK/QAM modulation is employed in an STBC scheme, we have a total of $M=\mathcal{M}^{V}$ legitimate STBC code matrix combinations for $V$ consecutive symbol periods. Based on the STBC's model of Eq. (1), the conditional probability of receiving a signal matrix $\mathbf{Y}$ given that an $M$-ary STBC code matrix $\mathbf{X}_{m}, m \in\{1, \ldots, M\}$, was transmitted over uncorrelated flat Rayleigh fading channels is determined by the PDF of the noise, which is given by:

$$
\begin{aligned}
& p\left(\mathbf{Y} \mid \mathbf{X}_{m}\right)=\frac{1}{\left(\pi N_{0}\right)^{V N_{r}}} \exp \left(\frac{-\left\|\mathbf{Y}-\mathbf{H} \mathbf{X}_{m}\right\|^{2}}{N_{0}}\right), \\
& =\prod_{v=1}^{V} \prod_{r=1}^{N_{r}} \frac{1}{\pi N_{0}} \exp \left(\frac{-\left|\mathbf{y}_{(r, v)}-\sum_{t=1}^{N_{t}} \mathbf{h}_{(r, t)} \mathbf{x}_{m(t, v)}\right|^{2}}{N_{0}}\right) .
\end{aligned}
$$

The channel capacity per symbol period evaluated for the STBC scheme when using complex-valued $\mathcal{M}$-ary PSK/QAM for transmission over the DCMC can be shown to be:

$$
\begin{gathered}
C_{\mathrm{DCMC}}^{\mathrm{STBC}}=\frac{1}{V} \max _{p\left(\mathbf{X}_{1}\right) \ldots p\left(\mathbf{X}_{M}\right)} \sum_{m=1}^{M} \int_{\mathbf{Y}} p\left(\mathbf{Y} \mid \mathbf{X}_{m}\right) p\left(\mathbf{X}_{m}\right) \\
\cdot \log _{2}\left(\frac{p\left(\mathbf{Y} \mid \mathbf{X}_{m}\right)}{\sum_{n=1}^{M} p\left(\mathbf{Y} \mid \mathbf{X}_{n}\right) p\left(\mathbf{X}_{n}\right)}\right) d \mathbf{Y}
\end{gathered}
$$

where the right hand side of Eq. (8) is maximized, when we have $p\left(\mathbf{X}_{m}\right)=1 / M$ for $m \in\{1, \ldots, M\}$. Hence, Eq. (8) can be simplified to:

$$
\begin{aligned}
C_{\mathrm{DCMC}}^{\mathrm{STBC}}= & \frac{\log _{2}(M)}{V}- \\
& \frac{1}{M V} \sum_{m=1}^{M} E\left[\log _{2} \sum_{n=1}^{M} \exp \left(\Psi_{m, n}\right) \mid \mathbf{X}_{m}\right]
\end{aligned}
$$




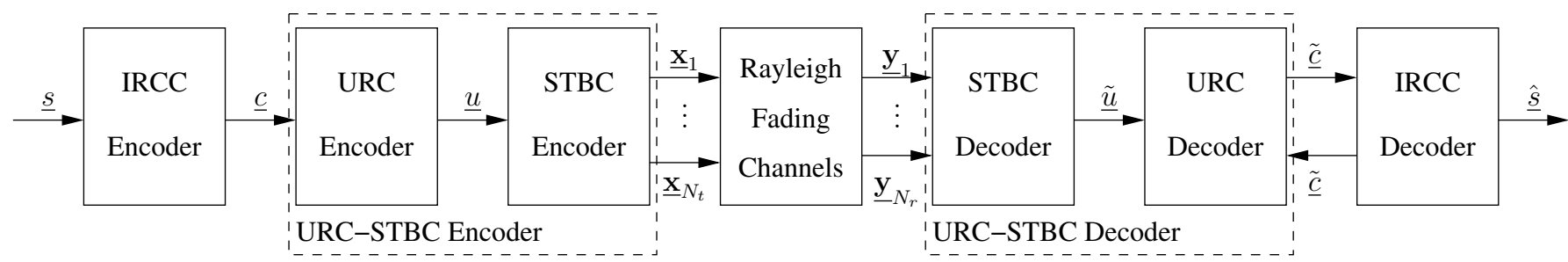

Fig. 1. The system block diagram of the IRCC-URC-STBC scheme. The notations $\underline{s}, \underline{c}, \underline{u}, \underline{\mathbf{x}}_{j}, \underline{\mathbf{y}}_{k}, \underline{\tilde{u}}, \underline{\tilde{c}}$ and $\underline{\hat{s}}$ denote the vectors of the input bits to IRCC encoder, the input bits to URC encoder, the input bits to the STBC encoder, the STBC coded symbols for transmitter $j$, the received symbols at receiver $k$, the extrinsic LLRs of STBC input bits, the extrinsic LLRs of URC input bits and the estimates of IRCC input bits, respectively. The bit-based interleavers between the IRCC and URC encoders/decoders are not shown for simplicity.

where $E\left[A \mid \mathbf{X}_{m}\right]$ is the expectation of $A$ conditioned on $\mathbf{X}_{m}$ and the expectation in Eq. (9) is taken over $\mathbf{H}$ and $\mathbf{W}$, while $\Psi_{m, n}$ is given by:

$$
\Psi_{m, n}=\frac{-\left\|\mathbf{H}\left(\mathbf{X}_{m}-\mathbf{X}_{n}\right)+\mathbf{W}\right\|^{2}+\|\mathbf{W}\|^{2}}{N_{0}} .
$$

Based on the DCMC capacity formula in Eq. (9) we can compute the capacity of any STBC scheme by substituting the corresponding STBC code matrix $\mathbf{X} \in \mathbb{C}^{N_{t} \times V}$ into Eq. (10).

The resultant bandwidth efficiency is computed by normalising the channel capacity given by Eq. (9), with respect to the product of the bandwidth $W$ and the signalling period $T$ :

$$
\eta=\frac{C}{W T}[\mathrm{bit} / \mathrm{s} / \mathrm{Hz}],
$$

where $W T=1$ for PSK/QAM schemes, when assuming zero Nyquist excess bandwidth. The bandwidth efficiency, $\eta$, is typically plotted against the SNR per bit given by: $E_{b} / N_{0}=\mathrm{SNR} / \eta$. For simplicity we will refer to $\eta$ as capacity.

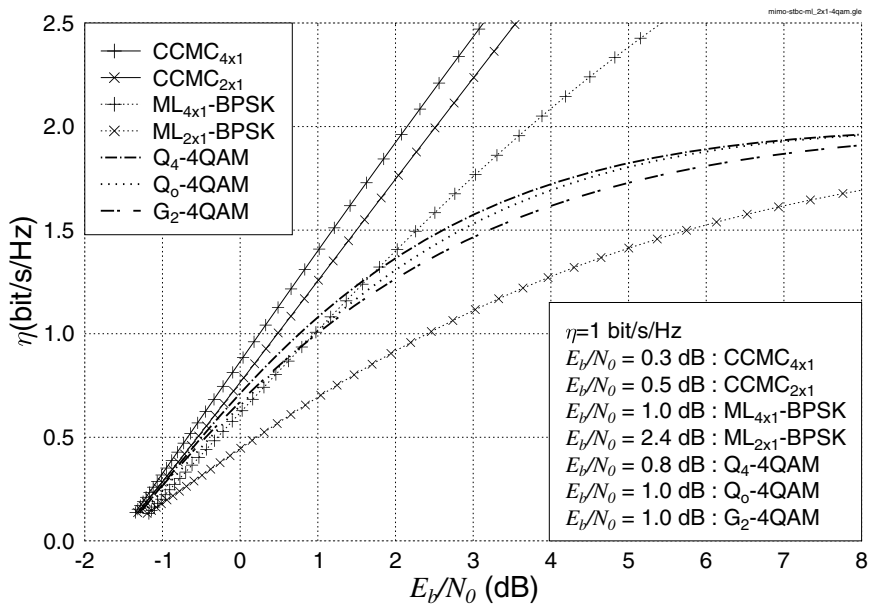

Fig. 2. The capacity of various STBC and ML schemes when communicating over uncorrelated Rayleigh fading channels using $N_{r}=1$ receive antenna.

We consider the scenario when $N_{r}=1$ receive antenna is used. The DCMC capacity curves of the three STBC codes mentioned in Section II-B are shown in Fig. 2 together with the DCMC capacity curves of the multiplexing-based MIMO scheme, which employed a Maximum Likelihood (ML) detector. The capacity curves of the unrestricted Continuous-input Continuous-output Memoryless Channel (CCMC) [15], [16] are also depicted in Fig. 2 for comparison. As shown in Fig. 2, the CCMC capacity recorded for for $N_{t}=4$ transmit antennas $\left(\mathrm{CCMC}_{4 \times 1}\right)$ is only marginally higher than that of the scheme using $N_{t}=2$ transmit antennas $\left(\mathrm{CCMC}_{2 \times 1}\right)$ due to the constraint of using a single receive antenna. Furthermore, for a fixed asymptotic DCMC capacity of $\eta=2 \mathrm{bit} / \mathrm{s} / \mathrm{Hz}$, the multiplexing-based MIMO scheme using $N_{t}=2$ and Binary-Phase Shift Keying (BPSK) modulation ( $\mathrm{ML}_{2 \times 1}$-BPSK) is lower than that of the $G_{2}$-based
STBC scheme employing 4QAM $\left(G_{2}-4 \mathrm{QAM}\right)$. When the number of transmit antennas is increased from $N_{t}=2$ to $N_{t}=4$, the DCMC capacity of the multiplexing-based MIMO scheme (ML $\mathrm{M}_{4 \times 1}$-BPSK) improved significantly, while the improvement of the STBC schemes ( $Q_{4}-4 \mathrm{QAM}$ and $\left.Q_{o}-4 \mathrm{QAM}\right)$ was rather marginal. The corresponding $E_{b} / N_{0}$ values required for maintaining a DCMC capacity of $\eta=$ $1 \mathrm{bit} / \mathrm{s} / \mathrm{Hz}$ for the various STBC schemes are tabulated in the second column of Table I.

\section{B. Code design}

We consider an average coding rate of $R=0.5$ for the IRCC outer code. Hence the effective throughput is $R \log _{2} 4=1$ bit when 4QAM modulation is employed by the full-rate STBC schemes.

According to the area properties [12], [17] of the EXIT charts, the area under the EXIT curve of the inner decoder is approximately equal to the channel capacity attained, when the channel input is uniformly distributed. Furthermore, the area under the EXIT curve of the outer code is approximately equal to $(1-R)$, where $R$ is the outer code rate. These properties were only proven for the scenario where the Binary Erasure Channel (BEC) [17] was used to model the $a$ priori probabilities. It was proven in [17] that if the a priori channel is a BEC, then the area property is valid for any communication channels. When the BEC is replaced by BPSK transmissions over an AWGN channel to model the a priori probabilities, the resultant EXIT function changed only slightly [17]. We assumed BPSK transmission over an AWGN channel to model the a priori probabilities in this work. The area property was experimentally shown to be valid for the AWGN, Rayleigh and multipath communication channels [12], [17] when the a priori channel was modeled by BPSK transmissions over an AWGN channel. Furthermore, we found that an intermediate URC changes only the shape but not the area under the EXIT curve of the inner code.

We divide the $I_{A}$ and $I_{E}$ values on the abscissa and ordinate axes of the EXIT charts by $I_{A, \max }=\log _{2}(\mathcal{M})=2$ so that the area of the EXIT chart is normalized to unity. Hence, the normalized area $A_{E}$ under the EXIT curve of the 4QAM-assisted URC-STBC inner decoder becomes $A_{E} \approx \eta / \log _{2}(\mathcal{M})=1 / 2=0.5$ at the specific $E_{b} / N_{0}$ value where achieving a capacity of $\eta=1 \mathrm{bit} / \mathrm{s} / \mathrm{Hz}$ becomes possible. The normalized EXIT curves of the various STBC schemes computed based on the soft-output of the various STBC decoders are plotted in Figs. 3, 4 and 5. Note that for a given $\mathcal{M}$-ary modulation aided STBC scheme employing $N_{t}$ number of transmit antennas and using $V=N_{t}$ symbol periods, we can detect the $N_{t}$ symbols using the following three methods:

1) jointly detect all $N_{t}$ symbols over $N_{t}$ consecutive symbol periods as a combined $\left[N_{t} \times \log _{2}(\mathcal{M})\right]$-bit symbol;

2) detect the $N_{t}$ symbols spanning $N_{t}$ symbol periods as $N_{t}$ number of $\log _{2}(\mathcal{M})$-bit symbols; or 


\begin{tabular}{|l|c|c|c||c|}
\hline Code $\left(N_{t} \mathrm{x} N_{r}\right)$ & $\eta=1$ & $A_{E}=0.5$ & Tunnel & IRCC $\left[\alpha_{1} z^{1}+\alpha_{2} z^{2}+\ldots+\alpha_{k} z^{k}+\ldots+\alpha_{17} z^{17}\right]$ \\
\hline \hline$G_{2}$-4QAM $(2 \mathrm{x} 1)$ & $1.0 \mathrm{~dB}$ & $1.0 \mathrm{~dB}$ & $1.3 \mathrm{~dB}$ & {$\left[0.0146889 z^{3}+0.475139 z^{6}+0.106222 z^{10}+0.117081 z^{12}+\right.$} \\
& & & & $\left.0.096298 z^{13}+0.019344 z^{16}+0.087791 z^{17}\right]$ \\
\hline$Q_{O}-4 \mathrm{Q} A M(4 \mathrm{x} 1)$ & $1.0 \mathrm{~dB}$ & $1.0 \mathrm{~dB}$ & $1.3 \mathrm{~dB}$ & {$\left[0.243488 z^{3}+0.126888 z^{5}+0.060988 z^{7}+0.075820 z^{8}+\right.$} \\
& & & & $\left.0.122799 z^{9}+0.198512 z^{13}+0.171530 z^{17}\right]$ \\
\hline$Q_{4}$-4QAM $(4 \mathrm{x} 1)$ & $0.8 \mathrm{~dB}$ & $0.8 \mathrm{~dB}$ & $1.1 \mathrm{~dB}$ & {$\left[0.169761 z^{2}+0.057011 z^{3}+0.076480 z^{4}+0.110251 z^{6}+\right.$} \\
& & & & $\left.0.108037 z^{8}+0.078073 z^{9}+0.212904 z^{13}+0.187505 z^{17}\right]$ \\
\hline$Q_{2}$-16QAM (2x1) & $3.0 \mathrm{~dB}$ & $3.0 \mathrm{~dB}$ & $3.4 \mathrm{~dB}$ & {$\left[0.372527 z^{5}+0.121322 z^{6}+0.208989 z^{10}+0.183134 z^{13}+\right.$} \\
& & & & $\left.0.114062 z^{17}\right]$ \\
\hline
\end{tabular}

TABLE I

THE TABLE of $E_{b} / N_{0}$ VALUES AND IRCC CODES. THE EXPONENT OF THE DUMMY VARIABLE $z$ DENOTES THE INDEX OF THE SUBCODES USED IN THE IRCC.

3) detect the $N_{t}$ symbols over $N_{t}$ symbol periods, as $N_{t} \times$ $\log _{2}(\mathcal{M})$ number of binary symbols.

Let us denote the combined symbol-oriented detection scheme based on method 1 using the subscript $s$ and that based on method 2 using the subscript $m$ to indicate $\mathcal{M}$-ary detection. We found that the EXIT curves of the $G_{2, s}$ and $G_{2, m}$ decoders are identical, as shown in Fig. 3. However, the EXIT curves of the $Q_{o, s}$ and $Q_{o, m}$ decoders as well as of the $Q_{4, s}$ and $Q_{4, m}$ decoders are different, as seen in Figs. 4 and 5, respectively. Note furthermore that the detection scheme based on method 1 involves detecting $2^{N_{t} \times \log _{2}(\mathcal{M})}$ number of levels for each $\left[N_{t} \times \log _{2}(\mathcal{M})\right]$-bit symbol, which is significantly more complex than the detection scheme based on method 2 involving the detection of $2^{\log _{2}(\mathcal{M})}$ number of levels for each $\log _{2}(\mathcal{M})$-bit symbol. We also found from Figs. 3, 4 and 5 that all STBC schemes are capable of maintaining an area of $A_{E}=0.5$ under the normalized EXIT chart at the corresponding $E_{b} / N_{0}$ capacity limit provided in Table I. Hence, the area property was verified also for STBC schemes.

As seen in Fig. 1, the soft-output of the STBC decoder is fed to the URC decoder. Note that when the EXIT curve of the STBC decoder is a slanted line, as in the case of $Q_{o, m}$ and $Q_{4, m}$, extrinsic information exchange using decoding iterations between the STBC decoder and the URC decoder are needed in order to achieve a near-capacity performance. However, in this contribution we aim for combining the STBC and URC decoders into an amalgamated STBCURC decoder, where no iterations are required between the STBC and URC decoders. An STBC-URC decoder can be realized with the aid of the symbol-based URC having $B$ bits for each symbol, where $B=\log _{2}(4)=2, B=4 \times \log _{2}(4)=8$ and $B=4 \times \log _{2}(4)=8$ for the schemes using the $G_{2, m}-4 \mathrm{QAM}, Q_{o, s}-4 \mathrm{QAM}$ and $Q_{4, s}-4 \mathrm{QAM}$ arrangements, respectively. The resultant EXIT curves recorded for the various STBC-URC schemes are shown in Figs. 3, 4 and 5. Since these EXIT curves are slanted, we need an outer code, which exhibits an EXIT curve matching that of the URC-STBC scheme. An ideal candidate for such an outer code is constituted by IRCCs [12].

\section{RESUlts AND Discussions}

We employed the 17 subcodes used in [12] in our IRCC design. In order to solve the EXIT curve-fitting problem of IRCC design, an iterative constituent code weight optimization solution was proposed in [11]. With the aid of this algorithm [11], the optimized weighting coefficients can be obtained. First, we compute the URC-STBC EXIT curve for an $E_{b} / N_{0}$ value, which is $0.1 \mathrm{~dB}$ higher than the corresponding $E_{b} / N_{0}$ value where the area under the curve is $A_{E}=0.5$. Then, we compute the optimized weighting coefficients of the IRCC in order to generate an EXIT curve for the outer IRCC that matches the EXIT curve of the inner URC-STBC. Once the best IRCC weights are found, they can be employed by the IRCC encoder. When there is an open EXIT chart tunnel leading to the $I_{E}=1.0$ value, decoding convergence to an infinitesimally low Bit Error Ratio (BER) value can be achieved [9]. The corresponding IRCC weights of each of the STBC-URC schemes are tabulated in Table I. We found from Figs. 3, 4 and 5 that open EXIT chart tunnels leading to the $I_{E}=1.0$ value may be obtained for $E_{b} / N_{0}$ values as close as 0.3 dB from the corresponding STBC-based DCMC capacity for our STBC-4QAM-URC-IRCC schemes. For example, the EXIT curve of the inner $G_{2, m}$-4QAM-URC decoder is recorded at $E_{b} / N_{0}=1.3 \mathrm{~dB}$ and there is an open EXIT chart tunnel leading to the $I_{E}=1.0$, value when employing the appropriately matched IRCC scheme shown in Fig. 3. The specific IRCC optimized for the $G_{2}$-4QAM-URC scheme invokes seven subcodes, where the weighting coefficients, $\alpha_{k}$, are non-zero for the subcodes of index $k=\{3,6,10,12,13,16,17\}$, which are shown in Table I. Note that the proposed $G_{2, m}-4 \mathrm{QAM}$ and $Q_{4, s}-4$ QAM assisted schemes perform within $\mathbf{0 . 8} \mathbf{~ d B}$ from the corresponding CCMC capacity.

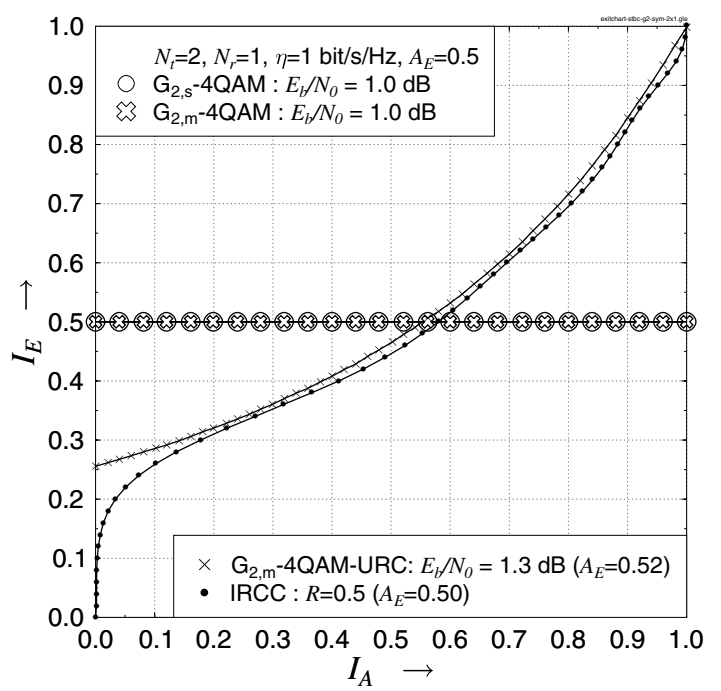

Fig. 3. Normalized EXIT charts for $G_{2}-4$ QAM, $G_{2, m}-4$ QAM-URC and IRCC when communicating over uncorrelated Rayleigh fading channels.

We have also designed a 16QAM scheme for attaining a throughput of $\eta=2 \mathrm{bit} / \mathrm{s} / \mathrm{Hz}$ using $G_{2}$ and $R=1 / 2$ IRCC scheme. At a capacity of $2 \mathrm{bit} / \mathrm{s} / \mathrm{Hz}$, the $E_{b} / N_{0}$ values required for $\mathrm{CCMC}_{2 \times 1}, \mathrm{ML}_{2 \times 1^{-}}$ 4QAM and $G_{2}$-16QAM are $2.6 \mathrm{~dB}, 3.3 \mathrm{~dB}$ and $3.0 \mathrm{~dB}$, respectively. Again, due to the employment of a single receive antenna, the $\mathrm{ML}_{2 \times 1}$-4QAM scheme exhibits a lower capacity compared to the $G_{2^{-}}$ 16QAM scheme, although both schemes have the same asymptotic capacity of $\eta=4 \mathrm{bit} / \mathrm{s} / \mathrm{Hz}$. A symbol-based URC having $\log _{2}(16)=$ 4-bit for each URC-encoded symbols is utilized. As shown in Fig. 6, the proposed $G_{2, m}$-16QAM-URC-IRCC scheme achieves a decoding convergence to an infinitesimally low BER at $3.4 \mathrm{~dB}$, which is only 0.4 dB from the DCMC capacity and $0.8 \mathrm{~dB}$ from the CCMC capacity. Two decoding trajectory snapshots of the $G_{2, m}$-16QAMURC-IRCC decoder, which are computed based on Monte-Carlo simulations using a block length of 100000 4-bit symbols are also shown in Fig. 6. The decoding trajectories traverse through the EXIT tunnel from the $\left(I_{A}=0, I_{E}=0\right)$ point to the $\left(I_{A}=1, I_{E}=1\right)$ at 


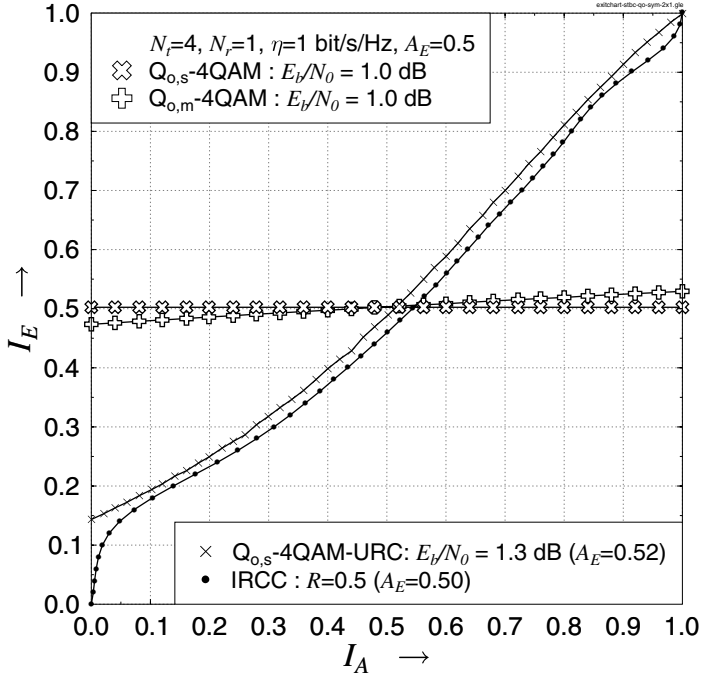

Fig. 4. Normalized EXIT charts for $Q_{o}-4 \mathrm{QAM}, Q_{o, s}-4 \mathrm{QAM}-\mathrm{URC}$ and and IRCC when communicating over uncorrelated Rayleigh fading channels.

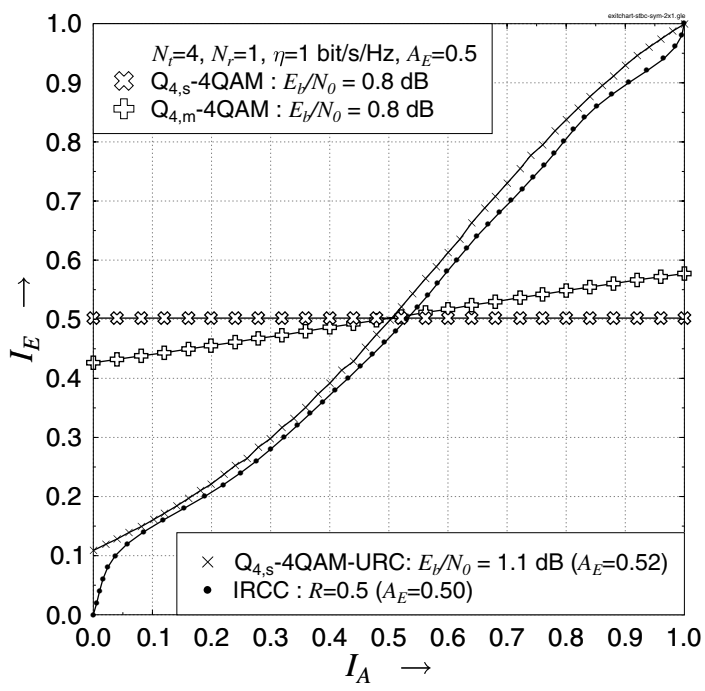

Fig. 5. Normalized EXIT charts for $Q_{4}-4 \mathrm{QAM}, Q_{4, s}-4 \mathrm{QAM}-\mathrm{URC}$ and and IRCC when communicating over uncorrelated Rayleigh fading channels.

$E_{b} / N_{0}=3.4 \mathrm{~dB}$.

\section{CONCLUSIONS}

In this contribution, we derived the DCMC capacity formula of STBC schemes based on the STBC code matrix. The performance of the STBC-assisted schemes was studied with the aid of EXIT chart analysis. Symbol-based URC schemes were utilized for assisting the STBC scheme for attaining near-capacity performance. IRCC schemes individually optimized for the various STBC-URC schemes were employed as outer codes. The proposed iteratively decoded STBC-URC-IRCC schemes are capable of having a decoding convergence at near-capacity $E_{b} / N_{0}$ values. The proposed schemes perform within $0.3 \mathrm{~dB}$ or $0.4 \mathrm{~dB}$ from the corresponding DCMC capacity. We note that it is beneficial to employ STBC schemes, when only a single receive antenna is employed, because the DCMC capacity of the STBC is higher than that of the corresponding spatial multiplexing based MIMO scheme using the same number of antennas and achieving the same throughput. The $G_{2}$ scheme using $\mathcal{M}$-QAM is the most computationally efficient one in terms of its decoding complexity, because it performed well, when detected based on $\log _{2}(\mathcal{M})$-bit symbols rather than based on the more complex detection of $\left[N_{t} \times \log _{2}(\mathcal{M})\right]$-bit symbols.

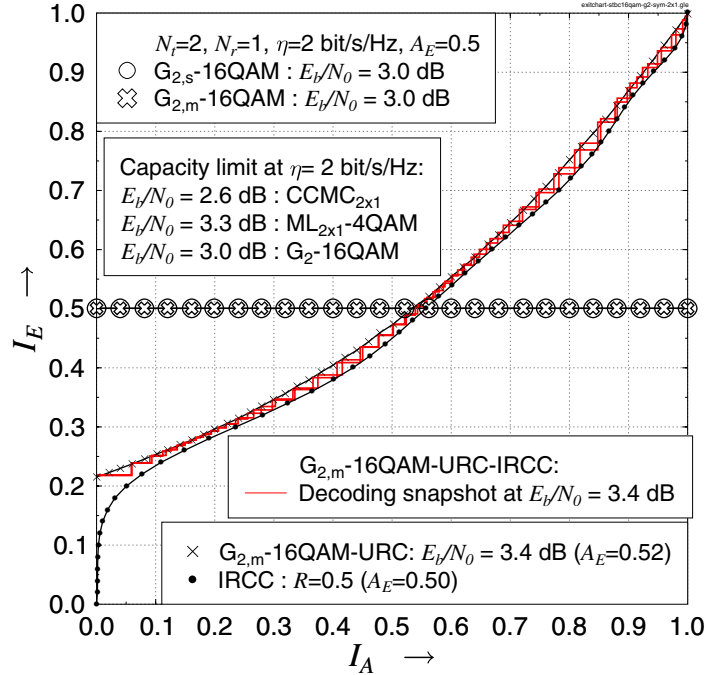

Fig. 6. Normalized EXIT charts for $G_{2}$-16QAM, $G_{2, m}-16 \mathrm{QAM}-\mathrm{URC}$ and and IRCC when communicating over uncorrelated Rayleigh fading channels.

\section{REFERENCES}

[1] G. Foschini Jr. and M. Gans, "On limits of wireless communication in a fading environment when using multiple antennas," Wireless Personal Communications, vol. 6, pp. 311-335, March 1998.

[2] E. Telatar, "Capacity of multi-antenna Gaussian channels," European Transactions on Telecommunication, vol. 10, pp. 585-595, Nov-Dec 1999.

[3] A. F. Naguib, V. Tarokh, N. Seshadri and A. R. Calderbank, "A spacetime coding modem for high-data-rate wireless communications," IEEE Journal on Selected Areas in Communications, vol. 16, pp. 1459 - 1478, October 1998.

[4] S. M. Alamouti, "A simple transmitter diversity scheme for wireless communications," IEEE Journal on Selected Areas in Communications, vol. 16, pp. 1451-1458, October 1998.

[5] V. Tarokh, H. Jafarkhani and A.R. Calderbank, "Space-time block codes from orthogonal designs," IEEE Transactions on Information Theory, vol. 45, pp. 1456-1467, July 1999.

[6] H. Jafarkhani, "A quasi-orthogonal space-time block code," IEEE Communications Letter, vol. 49, pp. 1-4, January 2001.

[7] L. He and $\mathrm{H}$. Ge, "A new full-rate full-diversity orthogonal space-time block coding scheme," IEEE Communications Letters, vol. 7, pp. 590592, December 2003.

[8] R. Calderbank, S. Das, N. Al-Dhahir and S. Diggavi, "Construction and analysis of a new quaternionic space-time code for 4 transmit antennas," Communications in Information and System: International Press, vol. 5, no. 1, pp. 97-122, 2005.

[9] S. ten Brink, "Convergence behaviour of iteratively decoded parallel concatenated codes," IEEE Transactions on Communications, vol. 49, pp. 1727-1737, October 2001.

[10] S. ten Brink, "Rate one-half code for approaching the Shannon limit by 0.1 dB," IEE Electronics Letters, vol. 36, pp. 1293-1294, July 2000.

[11] M. Tüchler and J. Hagenauer, "Exit charts and irregular codes," in Proceeding of the 36th Annual Conference on Information and System Sciences [CDROM], (Princeton, NJ, USA), March 2002.

[12] M. Tüchler, "Design of serially concatenated systems depending on the block length," IEEE Transactions on Communications, vol. 52, pp. 209218, February 2004.

[13] M. Tüchler, "Convergence prediction for iterative decoding of threefold concatenated systems," in GLOBECOM '02, vol. 2, (Taipei, Taiwan), pp. 1358-1362, 17-21 November 2002.

[14] S. X. Ng, J. Wang, M. Tao, L.-L. Yang and L. Hanzo, "Iteratively decoded variable-length space-time coded modulation: code construction and convergence analysis," IEEE Transactions on Wireless Communications, vol. 6, pp. 1953-1963, May 2007.

[15] J. G. Proakis, Digital Communications. Mc-Graw Hill International Editions, 3rd ed., 1995.

[16] S. X. Ng and Hanzo, "On the mimo channel capacity of multidimensional signal sets," IEEE Transactions on Vehicular Technology, vol. 55, pp. 528-536, March 2006.

[17] A. Ashikhmin, G. Kramer, and S. ten Brink, "Extrinsic information transfer functions: model and erasure channel properties," IEEE Transactions on Information Theory, vol. 50, pp. 2657-2673, November 2004. 